\title{
Simulating high-temperature superconductivity model Hamiltonians with atoms in optical lattices
}

\author{
Alexander Klein and Dieter Jaksch \\ Clarendon Laboratory, University of Oxford, Parks Road, Oxford OX1 3PU, United Kingdom
}

(Received 12 January 2006; published 26 May 2006)

\begin{abstract}
We investigate the feasibility of simulating different model Hamiltonians used in high-temperature superconductivity. We briefly discuss the most common models and then focus on the simulation of the so-called $t-J-U$ Hamiltonian using ultra-cold atoms in optical lattices. For this purpose, previous simulation schemes to realize the spin interaction term $J$ are extended. We especially overcome the condition of a filling factor of exactly one, which otherwise would restrict the phase of the simulated system to a Mott-insulator. Using ultra-cold atoms in optical lattices allows simulation of the discussed models for a very wide range of parameters. The time needed to simulate the Hamiltonian is estimated and the accuracy of the simulation process is numerically investigated for small systems.
\end{abstract}

DOI: 10.1103/PhysRevA.73.053613

PACS number(s): 03.75.Ss, 03.67.-a

\section{INTRODUCTION}

Since its discovery, high-temperature superconductivity [1] has attracted much attention on the theoretical as well as on the experimental side. Nevertheless, the mechanisms which lead to this effect are not completely understood [2]. On the theoretical side one encounters the problem that the physics of a very complex many-body system has to be described and calculated. Because quantum effects play a crucial role, the calculation of these systems is a very hard task on a classical computer. Furthermore it is not certain that the investigated theoretical models accurately describe the macroscopic features of superconducting materials used in experiments. Experimental efforts to test these models have suffered from difficulties in changing the system parameters for a given superconductor. In general different parameter sets require different types of superconducting material. Due to all these obstacles it is worthwhile searching for a versatile system which can be used to accurately simulate hightemperature superconductor models.

As we will show in this paper ultra-cold atoms in optical lattices are a very good candidate for simulating hightemperature superconductivity. During the past few years the field of cold atom physics has made tremendous progress and entered the regime of accurately controlled strongly correlated systems. Seminal experiments with bosonic atoms have, e.g., realized the Mott-insulator to superfluid transition and cold controlled collisions between atoms in optical lattices [3-6]. They found excellent agreement with corresponding theoretical models [7,8]. For clouds of fermionic atoms superfluidity has been demonstrated by creating vortices [9] and Fermi surfaces of atoms loaded into threedimensional optical lattices have been measured [10]. Optical lattice systems are also very flexible; a large range of Hamiltonians can be realized and system parameters are easily varied over a wide range by changing external laser parameters [11]. For instance, it has also been proposed to implement effective magnetic fields [12-17] and even the implementation of non-Abelian gauge fields seems to be feasible $[18,19]$.

We will briefly discuss some of the model Hamiltonians which are used to describe high-temperature superconductive cuprates. We will then focus on the $t-J-U$ Hamiltonian [20], which includes a spin-spin coupling term $J$ additionally to hopping $t$ and on-site interaction $U$. The simulation of this Hamiltonian can be used for obtaining a deeper understanding of the corresponding model, and also enlarges the parameter space by an additional, freely tunable interaction term $J$. In analogy to the cuprates we expect this model to show a very rich phase diagram for the atoms in the optical lattice including the appearance of (anti)ferromagnetic phases.

The simulation of the spin-spin interaction part in optical lattices has already been discussed in Refs. [21-23]. Atoms with two internal states simulate a spin chain and the spinspin interaction is implemented by state dependent shifting of the atoms causing controlled atomic collisions. These collisions induce state dependent phase shifts of the atomic wave function mimicking the spin-spin interaction. However, all of the proposals [21-23] require a filling of very close to or exactly one atom per lattice site. For superconductors, this corresponds to a Mott-insulating state. In order to extend this method to the simulation of the $t-J-U$ Hamiltonian we will relax the condition of having exactly one atom in each lattice site. Additional shifts of the atoms will be necessary for simulating the spin interaction term $J$ and we will also show that by choosing appropriate parameters it is possible to implement effectively attractive interaction terms $U<0$.

The simulation of the time evolution of the whole $t-J-U$ Hamiltonian is performed via a Trotter-Suzuki expansion [21,24-28] and we will consider the case of temperature $T$ $=0$ in our calculations. To be able to experimentally observe superconductivity effects using our simulation method the temperature of the optical lattice atoms will have to be lower than $k_{\mathrm{B}} T<0.02 t$ [29], where $k_{\mathrm{B}}$ is the Boltzmann constant. Such low temperatures are experimentally difficult to achieve. However, theoretical proposals for fault tolerant loading of fermionic atoms [30] into the lowest motional band of an optical lattice and phonon assisted side band cooling within this motional band [31] exist. These methods will be realizable with current and near future experimental techniques and enable achievement and control of temperatures sufficiently small for our purpose.

This work is organized as follows. In Sec. II we briefly discuss some of the model Hamiltonians used to describe high-temperature superconductivity in cuprates. In Sec. III 
we explain how one of these Hamiltonians, namely the $t-J-U$ model, can be simulated using ultra-cold atoms in optical lattices. The simulation process is numerically tested in Sec. IV and we conclude in Sec. V.

\section{MODEL HAMILTONIANS FOR HIGH-T SUPERCONDUCTORS}

In this section we briefly discuss some of the model Hamiltonians which are used to describe high-temperature superconductors [32-34]. The most basic microscopic description is given by the Hubbard model

$$
\hat{\boldsymbol{H}}_{t U}=-t \sum_{\langle i, j\rangle, \sigma} \hat{\boldsymbol{c}}_{i, \boldsymbol{\sigma}}^{\dagger} \hat{\boldsymbol{c}}_{j, \boldsymbol{\sigma}}+U \sum_{j} \hat{\boldsymbol{n}}_{j, \uparrow} \hat{\boldsymbol{n}}_{j, \downarrow}
$$

The operator $\hat{c}_{j, \sigma}^{\dagger}\left(\hat{c}_{j, \sigma}\right)$ creates (annihilates) an electron with spin $\sigma=\uparrow, \downarrow$ in lattice site $j$ and obeys the standard fermionic anticommutation relations, the number operator is denoted by $\hat{n}_{j, \sigma}=\hat{c}_{j, \sigma}^{\dagger} \hat{c}_{j, \sigma}$. This Hamiltonian describes electrons in the lowest Bloch band tightly bound to the lattice sites formed by the atoms of the solid. The electrons can tunnel from one lattice site to the nearest-neighbor site (indicated by the brackets $\langle i, j\rangle)$ with hopping energy $t$ and the repulsive onsite Coulomb interaction is given by $U$. Because of the Pauli exclusion principle for electrons and the single Bloch band assumption in Hamiltonian Eq. (1), there can be a maximum of one electron of each spin state in a single lattice site. Hence no on-site interaction term for electrons in the same spin state is included in the Hamiltonian.

The Hamiltonian Eq. (1) describes the behavior of the electron gas in two-dimensional (2D) layers. If the lattice sites are all occupied by a single electron the system behaves like a Mott-insulating antiferromagnet. By hole-doping of the cuprate the behavior of the gas changes considerably and for a critical doping and temperatures below $T_{c}$ the solid gets superconductive (see, e.g., $[29,35])$. In a real superconductor many of these two-dimensional layers are stacked on top of each other. The influence of these layers can be included by a small inter-layer tunneling strength [36].

Quantum cluster calculations give very strong evidence that the Hubbard Hamiltonian Eq. (1) is already a sufficient minimal model to describe the main properties of the phase diagrams of cuprates [37]. Nevertheless, these calculations suffer from finite size effects. The simulation of this Hamiltonian using ultra-cold fermionic atoms in an optical lattice is straightforward and can overcome this problem for sufficiently large lattices. It requires the loading of the atoms into the lattice and choosing the lattice parameters such that twodimensional layers are created. Details on which parameter range can be simulated are discussed in Sec. III A.

However, the Hubbard Hamiltonian Eq. (1) does not describe all properties encountered in a superconductor [38]. Other effective model Hamiltonians have been discussed trying to incorporate such experimentally observed effects. For example, it has been suggested [39] to introduce a nextnearest neighbor hopping term

$$
\hat{H}_{n n}=-t^{\prime} \sum_{\langle\langle i, j\rangle\rangle, \sigma} \hat{c}_{i, \sigma}^{\dagger} \hat{c}_{j, \sigma} .
$$

These terms can be realized in the simulation process by using very shallow optical lattices. In this case, however, the probability to excite atoms into higher Bloch bands increases and off-site interaction terms $U_{\text {os }}$ are important. Also it becomes more difficult to adjust all parameters in this Hamiltonian independently. Therefore, in this publication, we do not discuss the realization of this model Hamiltonian any further.

Another effective model is the so-called $t-J-U$ Hamiltonian, which has recently been used in connection with gossamer superconductivity $[20,40]$. It is given by

$$
\hat{H}=\hat{H}_{t U}+\hat{H}_{J}
$$

where

$$
\hat{H}_{J}=J \sum_{\langle i, j\rangle} \hat{\mathbf{S}}_{i} \cdot \hat{\mathbf{S}}_{j}
$$

is the spin interaction Hamiltonian of electrons in neighboring lattice sites. The spin operator $\hat{\mathbf{S}}_{j}$ is defined by its components

$$
\hat{\mathbf{S}}^{w}=\frac{1}{2} \sum_{\varsigma, \zeta} \hat{c}_{\varsigma}^{\dagger} \sigma^{w} \hat{c}_{\zeta},
$$

where $\sigma^{w}$ are Pauli matrices, $w=x, y, z$. For very large interaction strengths $U$ Hamiltonian Eq. (3) converges to the socalled $t-J$ model, where double-occupancy of a single lattice site is excluded. This Hamiltonian has also very often been used to describe the behavior of high- $T_{c}$ cuprates. In the following sections we will investigate in detail how the $t-J-U$ Hamiltonian can be simulated using atoms in optical lattices. Such simulations can help to understand and test features of the model, and also to investigate a larger parameter space which is likely to exhibit very interesting phase diagrams.

\section{SIMULATION OF THE $t-J-U$ MODEL WITH ATOMS IN OPTICAL LATTICES}

In this section we show how Hamiltonian Eq. (3) can be simulated by using ultra-cold atoms in optical lattices. First we explain how to implement the $t$ - $U$-Hamiltonian and the spin-interaction independently from each other. Then these Hamiltonians are combined to realize the full $t-J-U$ Hamiltonian.

\section{A. The hopping and interaction terms}

The Hamiltonian $\hat{H}_{t U}$, Eq. (1), is simulated using a threedimensional optical lattice where hopping along the $z$-direction is suppressed by high potential barriers [11]. The lattice is filled with ultra-cold fermionic atoms occupying the lowest motional Bloch band only and moving in planes parallel to the $x y$-plane. We restrict our considerations to one such 2D layer of the optical lattice. The two spin states of the electrons are represented by two long-living internal states of 
the atoms which we denote by $\sigma=\uparrow, \downarrow$. The Hamiltonian which describes the dynamics of fermionic atoms in the lattice is given by $\hat{H}_{t U_{s}}$, cf. Eq. (1), with the hopping constant $t$ and the interaction strength $U_{s}$. By an appropriate choice of the lattice constants the system parameters $t$ and $U_{s}$ can be tuned over a wide range [11] and using a Feshbach resonance it is possible to change the $s$-wave scattering length between the atoms which gives additional control over the interaction strength $U_{s}$.

However, we are not completely free in our choice of the lattice depths. In order to observe the superconducting phase in high- $T_{c}$ cuprates the temperature has to be lower than the critical temperature $T_{c}$, which is for high-temperature superconductors of the order of $0.02 t$ [29]. For our system with atoms in an optical lattice we expect the same behavior. We consider a lattice depth of $5 E_{\mathrm{R}}$ with $E_{\mathrm{R}}=\hbar^{2}(2 \pi)^{2} / 2 m \lambda^{2}$ the recoil energy, $m$ the mass of the atoms and $\lambda$ the wave length of the laser producing the lattice potential. In this case the hopping term $t$ is of the order of $0.07 E_{\mathrm{R}}$ and thus the superconducting phase will be observable for temperatures lower than approximately $0.0014 E_{\mathrm{R}} / k_{\mathrm{B}}$. For the fermionic species ${ }^{6} \mathrm{Li}$ trapped in a lattice with $\lambda=670 \mathrm{~nm}$ this corresponds to a temperature of $5 \mathrm{nK}$. In recent experiments with ${ }^{6} \mathrm{Li}$ temperatures of about $30 \mathrm{nK}$ have been reported [9]. The required lower temperatures can be reached for example with phonon side band cooling [30,31].

As an aside we note that in the case of bosonic atoms we have to assume a very high interaction strength $U_{\uparrow \uparrow}=U_{\downarrow \downarrow}$ between two atoms in the same state to realize the above Hamiltonian $\hat{H}_{t U_{s}}$ and replace anticommutator relations with commutator relations. This interaction has to be much larger than the interspecies interaction strength $U_{\uparrow \downarrow}=U_{s}$, the hopping constant $t$ and the spin-spin interaction strength $J$. In this case all states with two or more identical atoms in the same lattice sites can be discarded. However, a direct mapping to a fermionic system using a Jordan-Wigner transformation is only possible in one spatial dimension [41]. There are proposals for similar mappings in higher dimensions which require the addition of Majorana fermions to the system $[42,43]$. Although appealing from a theoretical point of view it is not clear how to realize such systems with atoms in optical lattices. Nevertheless, in the case of bosons the above conditions on the interaction strength lead to a rich phase diagram similar to models discussed in Ref. [23].

\section{B. Implementation of the spin-spin interaction}

In general, the van der Waals interaction between two atoms in neighboring lattice sites is not sufficient to realize the required ratios of $J, t$, and $U$. Lowering the lattice barriers such that there is a significant nearest neighbor interaction also causes problems since in this case it is more likely that higher Bloch bands are occupied. Hence the spin interaction $J$ has to be simulated indirectly. Methods for achieving this have already been detailed in Refs. [21-23]. These proposals require a filling factor of very close to or exactly one atom per lattice site which corresponds to half-filling of the electron system and thus a Mott-insulating phase [35].
Therefore, these proposals cannot be used to simulate superconductivity.

In order to circumvent this problem we extend the scheme proposed in Ref. [22]. Let us first consider the onedimensional spin-spin interaction in $z$-direction, i.e.,

$$
\hat{H}_{z z}=J_{z} \sum_{\langle i, j\rangle} \hat{S}_{i}^{z} \hat{S}_{j}^{z}=\frac{J_{z}}{4} \sum_{\langle i, j\rangle} \hat{n}_{\uparrow}^{i} \hat{n}_{\uparrow}^{j}+\hat{n}_{\downarrow}^{i} \hat{n}_{\downarrow}^{j}-\hat{n}_{\uparrow}^{i} \hat{n}_{\downarrow}^{j}-\hat{n}_{\downarrow}^{i} \hat{n}_{\uparrow}^{j} .
$$

This type of interaction between the atoms is realized by state selectively moving atoms. They are stored in very deep optical lattices such that any hopping is strongly suppressed, i.e., $t=0$. The atoms in, e.g., state $|\uparrow\rangle$ are shifted and overlapped for a certain time with their neighboring atoms to the left and to the right in state $|\downarrow\rangle$. The resulting atom-atom interaction leads to the desired phase shift as described in Ref. [8]. For illustration let us assume two lattice sites described by the Fock states $\left|n_{\uparrow}^{1}, n_{\downarrow}^{1} ; n_{\uparrow}^{2}, n_{\downarrow}^{2}\right\rangle$, where $n_{\sigma}^{j}$ is the number of atoms in state $|\sigma\rangle$ in the $j$ th lattice site. After the above shifting procedure this state evolves to

$$
\left|n_{\uparrow}^{1}, n_{\downarrow}^{1} ; n_{\uparrow}^{2}, n_{\downarrow}^{2}\right\rangle \rightarrow \exp \left(\mathrm{i} \chi\left(n_{\downarrow}^{1} n_{\uparrow}^{2}+n_{\uparrow}^{1} n_{\downarrow}^{2}\right)\right)\left|n_{\uparrow}^{1}, n_{\downarrow}^{1} ; n_{\uparrow}^{2}, n_{\downarrow}^{2}\right\rangle,
$$

where $\chi$ is the phase acquired during the collision process. This is exactly the action of the last two summands $-\hat{n}_{\uparrow}^{i} \hat{n}_{\downarrow}^{j}-\hat{n}_{\downarrow}^{i} \hat{n}_{\uparrow}^{j}$ of Eq. (6).

However, this process is not yet sufficient to implement the spin interaction in $z$-direction. In order to achieve our goal the spin state of the atoms in every second lattice site has to be flipped, i.e., the operation $V_{\mathrm{fl}}=\sigma_{1}^{x} \otimes 1_{2} \otimes \sigma_{3}^{x} \otimes 1_{4}$ $\otimes \cdots$ has to be applied to the atoms. This operation requires addressing each second lattice site which can be done by using an additional standing wave laser field. The resulting superlattice must have twice the wavelength of the original lattice and its intensity minima need to coincide with every second lattice site of the trapping potential [44]. Thus the energy levels of every second atom are ac-Stark shifted out of resonance such that a microwave or laser-field driving the transition $|\uparrow\rangle \leftrightarrow|\downarrow\rangle$ realizes $V_{\mathrm{fl}}$.

By repeating this shifting process after the spin flip operation $V_{\mathrm{fl}}$ and flipping the atoms back the state evolves according to

$$
\left|n_{\uparrow}^{1}, n_{\downarrow}^{1} ; n_{\uparrow}^{2}, n_{\downarrow}^{2}\right\rangle \rightarrow \exp \left(-\mathrm{i} \chi\left(n_{\downarrow}^{1} n_{\downarrow}^{2}+n_{\uparrow}^{1} n_{\uparrow}^{2}\right)\right)\left|n_{\uparrow}^{1}, n_{\downarrow}^{1} ; n_{\uparrow}^{2}, n_{\downarrow}^{2}\right\rangle,
$$

where the collision time has to be chosen such that the acquired phase is equal to $-\chi$. The whole process induces dynamics according to Hamiltonian Eq. (6), where $\chi=J_{z} \tau / 4 \hbar$ and $\tau$ is the time for which $\hat{H}_{z z}$ is applied (details on calculating the phases can be found in Appendix A).

The creation of such spin-dependent phases has already been demonstrated experimentally. In Ref. [5] the authors used $\mathrm{Rb}$ atoms in an optical lattice with $V_{0}=34 E_{\mathrm{R}}$. Excitations of atoms into higher Bloch bands were avoided by using an appropriate time of $\tau_{s}=40 \mu$ s to shift the atoms spin-dependently into the neighboring lattice sites [6]. After holding the atoms in the shifted position for $\tau_{h}=450 \mu \mathrm{s}$ and shifting them back again collisional phases of $\chi \approx 2 \pi$ were achieved. 
With being able to implement Hamiltonian Eq. (6) it is also possible to realize the Hamiltonians

$$
\begin{aligned}
& \hat{H}_{x x}=J_{x} \sum_{\langle i, j\rangle} \hat{S}_{i}^{x} \hat{S}_{j}^{x}, \\
& \hat{H}_{y y}=J_{y} \sum_{\langle i, j\rangle} \hat{S}_{i}^{y} \hat{S}_{j}^{y} .
\end{aligned}
$$

We first observe that with suitable laser pulses it is possible to implement the following rotations on single atoms

$$
\begin{gathered}
V_{y}=\exp \left(i \frac{\pi}{4} \sigma^{y}\right), \\
V_{x}=\exp \left(-i \frac{\pi}{4} \sigma^{x}\right) .
\end{gathered}
$$

By simultaneously applying one of these rotations on all atoms, implementing Hamiltonian Eq. (6) and applying the Hermitian conjugate of the same rotation we get the time evolutions $[21,22,45]$

$$
\begin{aligned}
& V_{y}^{\dagger} \exp \left(-\mathrm{i} \hat{H}_{z z} \frac{\tau}{\hbar}\right) V_{y}=\exp \left(-\mathrm{i} \hat{H}_{x x} \frac{\tau}{\hbar}\right), \\
& V_{x}^{\dagger} \exp \left(-\mathrm{i} \hat{H}_{z z} \frac{\tau}{\hbar}\right) V_{x}=\exp \left(-\mathrm{i} \hat{H}_{y y} \frac{\tau}{\hbar}\right) .
\end{aligned}
$$

The interaction strengths $J_{x}, J_{y}$, and $J_{z}$ can be tuned independently from each other by the choice of the collisional phase $\chi$ during the simulation of the respective Hamiltonian.

\section{Combining the Hamiltonians}

We simulate Hamiltonian Eq. (3) using the well known Trotter-Suzuki expansion [21,24-28] described in detail in Appendix B. In this approach the different parts of the Hamiltonian are simulated for a small time $\tau$ separately. For instance, in first order, the decomposition of the $t-J-U$ Hamiltonian reads

$$
\begin{aligned}
\exp \left(\frac{-\mathrm{i} \hat{H} \tau}{\hbar}\right)= & \exp \left(\frac{-\mathrm{i} \hat{H}_{z z} \tau}{\hbar}\right) V_{x}^{\dagger} \exp \left(\frac{-\mathrm{i} \hat{H}_{z z} \tau}{\hbar}\right) V_{x} \\
& \times V_{y}^{\dagger} \exp \left(\frac{-\mathrm{i} \hat{H}_{z z} \tau}{\hbar}\right) V_{y} \exp \left(\frac{-\mathrm{i} \hat{H}_{t U_{s}} \tau}{\hbar}\right) \\
& +O\left(\tau^{2}\right) .
\end{aligned}
$$

The last part $\exp \left(-\mathrm{i} \hat{H}_{t U_{S}} \tau / \hbar\right)$ of this time evolution is simulated by choosing a suitable lattice depth such that the required values of $t$ and $U_{s}$ are realized. After waiting a time $\tau$ [46] the lattice has to be ramped up avoiding any excitation into higher Bloch bands. Then the shifting and flipping processes $V_{\mathrm{fl}}$, as described in the previous section, have to be applied in order to simulate the time evolution of $\hat{H}_{z z}, \hat{H}_{y y}$, and $\hat{H}_{x x}$. The latter two require the implementation of the operations $V_{x}$ and $V_{y}$, cf. Eqs. (11) and (12). By repeating all of these simulation steps $m$ times a time $m \tau$ is simulated with an error $\propto \tau^{2} m$. Better accuracies can be achieved by using higher order approximations, see Appendix B.

During the simulation of the spin-spin interaction term two atoms may occupy the same lattice site $j$. This gives rise to an additional term not considered so far. We illustrate this by considering shifts in $x$-direction during the simulation of the spin interaction. The atoms will acquire an additional phase (compare Appendix A)

$$
\phi=K \int_{-\tau_{0}}^{\tau_{0}} \mathrm{~d} \tau \exp \left(-\frac{\left[x_{\uparrow}^{j}(\tau)-x_{\downarrow}^{j}(\tau)\right]^{2}}{2 x_{0}^{2}}\right),
$$

with $K$ given in Eq. (A3). This phase corresponds to an (additional) effective interaction term

$$
\hat{H}_{U}^{\mathrm{eff}}=U_{\mathrm{eff}} \sum_{j} \hat{n}_{j, \uparrow} \hat{n}_{j, \downarrow}
$$

where the interaction constant is given by $U_{\text {eff }}=\hbar \phi / \tau$. Note that this effective interaction does not diverge for $\tau \rightarrow 0$ as in this case also $\phi$ goes to zero. This interaction provides an additional opportunity for tuning the simulated interaction constant $U$. The total interaction for small $U_{\text {eff }} \ll 2 \pi \hbar / \tau$ is given by $U=U_{s}+3 d U_{\text {eff }}$ with $d$ the number of spatial dimensions in which the spin-spin interaction is simulated. Here the factor of 3 in front of the dimension number $d$ arises because the phase $\phi$ occurs in the simulations of $\hat{H}_{x x}, \hat{H}_{y y}$, and $\hat{H}_{z z}$ which are all simulated for the same time $\tau$. By an appropriate choice of $U_{s}$ and $\phi$ it is possible to simulate effective interactions which are attractive. For this purpose, the phase $\phi$ has to be chosen in such a way that it can be written as $\phi=2 \pi+U_{\text {eff }}^{\prime} \tau / \hbar$ with a small negative $U_{\text {eff }}^{\prime}$ and $2 \pi \gg\left|U_{\text {eff }}^{\prime}\right| \tau / \hbar$. The total simulated interaction is then given by $U=U_{s}+3 d U_{\text {eff }}^{\prime}$. For not too large $U_{s}$ this leads to a negative $U$ [47].

The total time for which the system can be simulated is restricted by the life- and decoherence-time of the ultra-cold atoms in the optical lattice. This is typically on the order of one second [3]. Hence we have to estimate the time which is needed for simulating one time step of Hamiltonian Eq. (3). During the shifting process heating of the atoms has to be avoided. Since the lattice is required to be very deep during the atoms are shifted hopping is strongly suppressed. This leads to a flat Bloch band and thus no excitation can take place within the band during the shift. Any heating thus means that atoms are excited into higher Bloch bands. In order to avoid such excitations we require for each shift of atoms in, e.g., state $|\uparrow\rangle$ into the neighboring lattice site a time larger than the inverse of the lattice site trapping frequency $\omega_{t}=\sqrt{V_{0} / E_{\mathrm{R}}} \hbar(2 \pi)^{2} / m \lambda^{2}$, as already demonstrated experimentally in Refs. $[5,6]$. Furthermore, the atoms have to be held in the shifted position for a certain time in order to achieve the desired phase. This phase is either $\chi$ or $2 \pi-\chi$. Thus the average time for one shifting and holding process is given by 


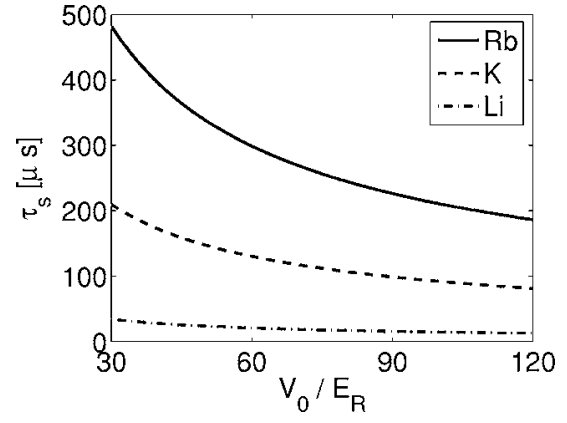

FIG. 1. Lower bounds for the calculated shifting and holding times $\tau_{s h}$ [cf. Eq. (18)] for lattices with ${ }^{87} \mathrm{Rb}$ atoms $(\lambda=826 \mathrm{~nm}$, $\left.a_{s}=5.1 \mathrm{~nm}\right),{ }^{40} \mathrm{~K}$ atoms $\left(\lambda=826 \mathrm{~nm}, a_{s}=5.5 \mathrm{~nm}\right)$ and ${ }^{6} \mathrm{Li}$ atoms $\left(\lambda=670 \mathrm{~nm}, a_{s}=2.4 \mathrm{~nm}\right)$. The values given in brackets are typical values used for the calculation of $\tau_{s h}$.

$$
\tau_{s h}>2 \pi\left(\frac{4}{\omega_{t}}+\frac{1}{K}\right) .
$$

The minimal shifting times for varying lattice depths are shown in Fig. 1 and are of the order of a few hundred microseconds for $\mathrm{Rb}$ or a few tens of microseconds for $\mathrm{Li}$. To simulate the time evolution of $\hat{H}_{z z}$ these shifts have to be repeated $2 d$ times.

The constraints for the ramping time between the application of the $t$ - $U$-Hamiltonian and the spin-spin interaction are twofold: The ramping has to be adiabatic on a time scale given by $1 / \omega_{t}$ to avoid excitations into higher Bloch bands and it has to be rapid compared to $\hbar \pi / 2 t$. This ensures that the system does not evolve for too long with a hopping term $t$ that is different from the desired one or even follows the change in $t$ adiabatically in contrast to the sudden change required by the Trotter-Suzuki expansion. The first constraint has been experimentally tested for $\mathrm{Rb}$ atoms [4] and was found to be fulfilled for ramping times longer than $50 \mu \mathrm{s}$ in accordance with theoretical calculations [48]. For typical lattice parameters the hopping time is given by $\hbar \pi / 2 t=1 \mathrm{~ms}$ and is thus more than one order of magnitude larger than the minimal ramping time required to avoid excitations into higher Bloch bands. Therefore, both constraints can be fulfilled at the same time. Furthermore, the ramping process conserves quasi-momentum, and excitations that do not change the quasi-momentum involve at least two hopping processes. Because the lattice is ramped on a time scale short compared to $\hbar \pi / 2 t$ excitations within the lowest Bloch band are, therefore, also strongly suppressed. Together with the time needed to simulate the $t$ - $U$-Hamiltonian it is thus feasible to simulate several hundreds of Trotter steps $m$ within the lifetime of the atoms in the lattice.

We finally remark that this procedure for simulating the $t$ - $J$ - $U$ Hamiltonian is compatible with the additional simulation of a magnetic field. Various methods for creating effective magnetic fields at the same time as the hopping and interaction terms in $\hat{H}_{t U}$ were recently proposed [12-17]. Thus it is possible to extend our setup for studying the $t-J-U$ Hamiltonian in external magnetic fields.

\section{Measuring the properties of the atom gas}

The properties of the time-evolved state can be probed by measurements of first- and second-order correlation func- tions as proposed in Ref. [49]. For this purpose a multiple matter-wave version of the Hanbury Brown and Twiss experiment [50] has to be realized. The atoms are released from their trapping potential and imaged after a certain time of flight. Depending on the phase of the atomic gas these images reveal interference patterns; second-order correlation functions give additional information on the phase and the underlying structure of the lattice. Such measurements have already been performed for bosonic atoms in the Mottinsulating phase $[3,51]$ and agree very well with the theoretical predictions [52]. With these techniques it will be possible to check the phase diagrams of the simulated systems and compare them to calculated or measured phase diagrams for different high-temperature superconductors (see, e.g., $[29,35,53])$.

\section{NUMERICAL SIMULATIONS}

As already mentioned in the Introduction the simulation of Hamiltonian Eq. (3) is a very hard computational task. Even for a few lattice sites an exact simulation of the system is no longer feasible and one has to use approximations such as mean-field theories or quantum Monte Carlo calculations. Since we want to compare the results of the simulation of Hamiltonian Eq. (3) with exact results we have thus restricted ourselves to the one-dimensional case with a few lattice sites only.

The anti-fidelity of the simulated state compared to a state which is time-evolved using the full Hamiltonian Eq. (3) is calculated as follows. Let $\hat{\mathcal{U}}_{0}(\tau)=\exp (-\mathrm{i} \hat{H} \tau / \hbar)$ be the full time evolution operator, $\hat{\mathcal{U}}(\tau)$ the simulated time evolution, $|\psi\rangle_{\text {in }}$ an input state and $|\psi\rangle=\hat{\mathcal{U}}(\tau)|\psi\rangle_{\text {in }},\left|\psi_{0}\right\rangle=\hat{\mathcal{U}}_{0}(\tau)|\psi\rangle_{\text {in }}$. Due to the Cauchy-Schwarz inequality and the properties of the matrix norm we get

$$
\begin{aligned}
& \left\|\hat{\mathcal{U}}(\tau)-\hat{\mathcal{U}}_{0}(\tau)\right\|^{2} \geqslant\left|\left(\left\langle\psi\left|-\left\langle\psi_{0}\right|\right)\left(|\psi\rangle-\left|\psi_{0}\right\rangle\right)\right|\right.\right. \\
& \quad=\left|2-2 \operatorname{Re}\left(\left\langle\psi \mid \psi_{0}\right\rangle\right)\right| .
\end{aligned}
$$

Since $\left|\left\langle\psi \mid \psi_{0}\right\rangle\right| \leqslant 1$ the rhs of Eq. (19) is always positive even without the modulus and we can rearrange

$$
\left|\left\langle\psi \mid \psi_{0}\right\rangle\right| \geqslant 1-\frac{1}{2}\left\|\hat{\mathcal{U}}(\tau)-\hat{\mathcal{U}}_{0}(\tau)\right\|^{2} .
$$

For $\left\|\hat{\mathcal{U}}(\tau)-\hat{\mathcal{U}}_{0}(\tau)\right\|^{2} \leqslant 2$ this yields an upper bound for the anti-fidelity $\mathcal{F}$

$$
\mathcal{F}=1-\left|\left\langle\psi \mid \psi_{0}\right\rangle\right|^{2} \leqslant\left\|\hat{\mathcal{U}}(\tau)-\hat{\mathcal{U}}_{0}(\tau)\right\|^{2}
$$

which can be easily calculated from the time evolution operators.

We have calculated this anti-fidelity $\mathcal{F}$ for several cases. First, we investigate a parameter set which gives a negative (attractive) interaction strength $U$ and compare this to the exact time evolution according to Eq. (3). Upper bounds for the corresponding anti-fidelity $\mathcal{F}$ are shown in Fig. 2. It has been assumed that no errors such as unprecise creation of the phase shifts $\chi$ or excitation of higher Bloch bands occur during the shifting process. For small times the slopes of the 


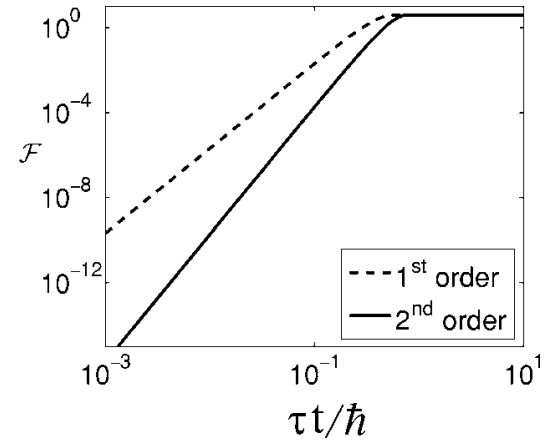

FIG. 2. Simulation of Hamiltonian Eq. (3) using first- and second-order Trotter expansions, cf. Eqs. (B1) and (B2). Upper bounds for the anti-fidelity $\mathcal{F}$ are shown. The number of lattice sites is $M=5$, the parameters for the simulation are $J=0.3 t, U_{s}=5 t$, $U_{\text {eff }}^{\prime}=-2 t, m=1$ leading to $U=-t$.

curves for the first- and second-order approximations agree very well with the predictions from first- and second-order expansions, cf. Eqs. (B1) and (B2). Note that the slopes shown are increased by a factor of two because we take the square of the matrix norm in order to calculate the antifidelity $\mathcal{F}$. Figure 2 shows that for small times the simulation of the time evolution of Hamiltonian Eq. (3) agrees very well with the full time evolution but the desired simulation times of order $100 \hbar / t$ can only be reliably achieved by repeating this simulation process.

The dependence of the upper bounds of the anti-fidelity $\mathcal{F}$ on the number $m$ of Trotter steps for a fixed time $\tau=100 \hbar / t$ is shown in Fig. 3 for values corresponding to typical parameters in high-temperature superconductivity [54]. As expected the fidelity gets better for an increasing number of steps. In second-order approximation for $m=500$ steps the anti-fidelity is already smaller than $10^{-3}$. For the same accuracy using the first-order approximation $m=900$ Trotter steps have to be used. This means in first-order approximation the lattice has to be ramped up and down 900 times and $\hat{H}_{z z}$ has to be simulated 2700 times with 1800 applications of $V_{x, y}$. In second order each single step is more complicated, but by choosing the simulation process in a judicious way only 500 rampings of the lattice are required. By optimizing the process (cf. Appendix B) only 2500 simu-

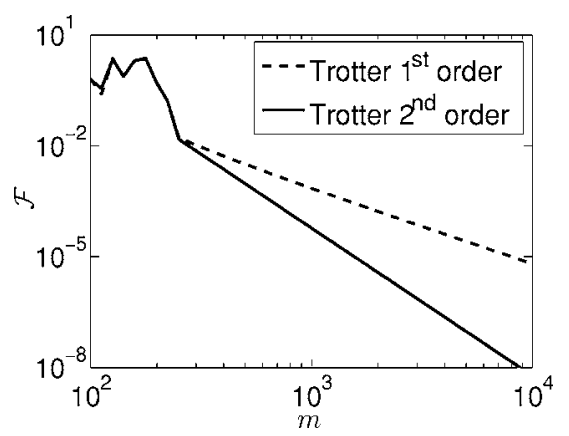

FIG. 3. Upper bounds for the anti-fidelity $\mathcal{F}$ for first- and second-order Trotter expansions, cf. Eq. (B4). The simulated time is fixed to $\tau=100 \hbar / t$, the number of lattice sites is $M=5$. We have used $J=0.3 t, U_{s}=10 t$, and $\phi=0$.

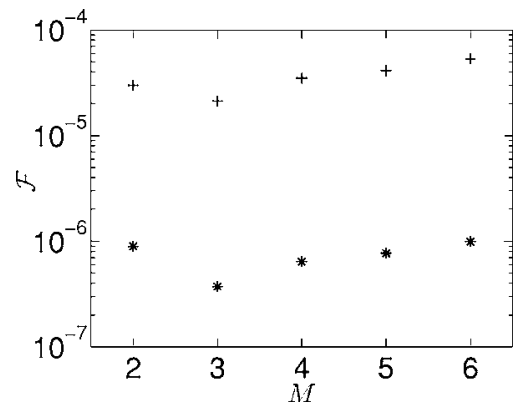

FIG. 4. Upper bounds for the anti-fidelity $\mathcal{F}$ vs number of lattice sites $M$ for first- (pluses) and second-order (stars) Trotter expansion. The simulated time was $\tau=0.01 \hbar / t$ and we have chosen $J=0.3 t$, $U_{s}=10 t$, and $\phi=0$.

lations of $\hat{H}_{z z}$ are required with 1500 applications of $V_{x, y}$.

To get an impression on how the anti-fidelity $\mathcal{F}$ scales with the number of lattice sites $M$ we calculated it for different values of lattice sites $M$ in a one-dimensional (1D) lattice. The results are shown in Fig. 4 for a simulated time of $\tau=0.01 \hbar / t$. The results indicate that the anti-fidelity $\mathcal{F}$ does not increase quickly with the number of lattice sites. Therefore, it should be possible to experimentally simulate systems of realistic size.

Since the time evolution of the full Hamiltonian Eq. (3) is only simulated approximately in our scheme, the state vector of the atoms after the time evolution will also only approximate the real state. However, we expect errors due to this effect to be small, since the necessary measurements to distinguish the phases involve only first- and second-order correlation functions. The influence of small imperfections of the simulated Hamiltonian on these functions will in general be smaller than the influence on the state vector estimated by the upper bounds of the anti-fidelity $\mathcal{F}$. Thus the deviations of the simulated phase diagrams is expected to be smaller than implied by the anti-fidelities.

\section{CONCLUSION}

In the present paper we have shown that it is feasible to simulate different model Hamiltonians which are used to describe high-temperature superconductive cuprates. The simulation of the most minimal model, the Hubbard model Eq. (1), can already be expected to deepen our understanding of the phase diagrams encountered in the cuprates. Since this model does not describe all experimentally measured effects and to enlarge the accessible parameter space which can be investigated using atoms in optical lattices we have especially discussed how to simulate the $t-J-U$ Hamiltonian Eq. (3), which requires the inclusion of an additional spin-spin interaction term $J$.

In order to make the simulation possible we have extended earlier proposals to create an effective spin interaction between atoms to the case where the lattice is not fully occupied. We found that near future technology will allow one to simulate Hamiltonian Eq. (3) for times which are longer than the typical time scales associated with the dynamics of the $t-J-U$ Hamiltonian. Hence the properties of the Hamil- 
tonian can be made visible using our simulation method. We also showed that by an appropriate choice of the lattice parameters and shifting times it is possible to create attractive on-site interactions $U$. Also, our method is compatible with the simulation of magnetic fields in the lattice.

Furthermore, we simulated the simulation process numerically for small systems and compared the results with exact calculations of the time evolution. For these simulations the results show very good agreement between the simulated and the real time evolution. Anti-fidelities $\mathcal{F}<10^{-3}$ can be achieved. Because of these results and of the experimental progress we are confident that the proposed method will help to deepen our knowledge and understanding of the $t-J-U$ Hamiltonian model.

\section{ACKNOWLEDGMENTS}

A.K. thanks Stephen R. Clark for useful discussions and acknowledges financial support from the Keble Association. This work was supported by the EPSRC (UK) through QIP IRC (GR/S82176/01) and project EP/C51933/1. The research was also supported by the EU through the STREP project OLAQUI (http://olaqui.df.unipi.it/).

\section{APPENDIX A: CALCULATION OF THE COLLISIONAL PHASES}

The exact phase shift due to the shift and interaction process can be calculated analytically [8]. If we assume deep lattice potentials of $V_{x, y, z}$, we can approximate the wave functions of the atoms located in one lattice site by Gaussians. For illustration we assume an atom of state $|\uparrow\rangle$ in lattice site $j$ and one of state $|\downarrow\rangle$ in lattice site $j+1$. The shift shall occur in $x$-direction. Let $x_{\uparrow}^{j}(\tau), x_{\downarrow}^{j+1}(\tau)$ denote the timedependent $x$-coordinates of the atoms. Because of the collision process the state of the two atoms will evolve to

$$
|1,0 ; 0,1\rangle \rightarrow \mathrm{e}^{-\mathrm{i} \chi}|1,0 ; 0,1\rangle
$$

with the collisional phase given by

$$
\chi=K \int_{-\tau_{0}}^{\tau_{0}} \mathrm{~d} \tau \exp \left(-\frac{\left[x_{\uparrow}^{j}(\tau)-x_{\downarrow}^{j+1}(\tau)\right]^{2}}{2 x_{0}^{2}}\right) .
$$

Here we have defined

$$
K=\frac{4 \pi a_{s} \hbar}{m} \frac{(\sqrt{2 \pi})^{3}}{\lambda^{3}}\left(\frac{V_{0}}{E_{\mathrm{R}}}\right)^{3 / 4},
$$

where $a_{s}$ is the $s$-wave scattering length between the two atoms in states $|\uparrow\rangle$ and $|\downarrow\rangle, V_{0}$ is the depth of the lattice,
$x_{0}=\sqrt[4]{E_{\mathrm{R}} / V_{0}} \lambda / 2 \pi$ and $E_{\mathrm{R}}=\hbar^{2}(2 \pi)^{2} / m \lambda^{2}$ is the recoil energy, $m$ the mass of the atoms and $\lambda$ the wavelength of the lasers creating the optical lattice. The time $\tau_{0}$ is chosen such that the entire shift process is contained in the time interval from $-\tau_{0}$ to $\tau_{0}$. For typical experimental parameters [5] of $V_{0}$ $=34 E_{\mathrm{R}}$ and ${ }^{87} \mathrm{Rb}$ atoms with $a_{s}=5.1 \mathrm{~nm}$ the prefactor in front of the integral has a value of roughly $K=19 \mathrm{rad} / \mathrm{ms}$ such that after a collisional time of a few hundred microseconds phase shifts of the order of $2 \pi$ can be achieved. For Li atoms the prefactor has a value of the order of $K=230 \mathrm{rad} / \mathrm{ms}$ and hence only roughly a tenth of the time is necessary to achieve similar phase shifts. The exact phase shifts depend on the details of the shifting process itself, i.e., on the exact form of the functions $x_{\sigma}^{j}(\tau)$.

\section{APPENDIX B: THE TROTTER-SUZUKI EXPANSION}

For an arbitrary Hamiltonian $\hat{H}_{\text {arb }}=\Sigma_{j=1}^{f} \hat{H}_{j}$ the TrotterSuzuki expansion $[21,24-28]$ is found by defining

$$
\begin{gathered}
Q_{1}(x)=\prod_{j=1}^{f} \exp \left(x \hat{H}_{j}\right), \\
Q_{2}(x)=\mathrm{e}^{\hat{H}_{1} x / 2} \cdots \mathrm{e}^{\hat{H}_{f-1} x / 2} \mathrm{e}^{\hat{H}_{f} x} \hat{\mathrm{H}}_{f-1} \hat{H}^{x / 2} \cdots \mathrm{e}^{\hat{H}_{1} x / 2},
\end{gathered}
$$

where $x=-i \tau / \hbar$ and $\tau$ is the simulation time. The operators $Q_{1}(x)$ and $Q_{2}(x)$ are approximations to the time evolution operator of $\hat{H}_{\text {arb }}$ with

$$
\exp \left(\hat{H}_{\mathrm{arb}} x\right)=Q_{1}(x)+O\left(|x|^{2}\right)=Q_{2}(x)+O\left(|x|^{3}\right) .
$$

Higher order approximations to the time evolution of $\hat{H}_{\text {arb }}$ exist, but they either require the simulation of negative time which is difficult for the hopping Hamiltonian $\hat{H}_{t U}$, or they include terms which make the simulation unstable [25]. Therefore, we simulate longer times using a generalized version of the Trotter formula [28] with $m$ simulation steps, reading

$$
\exp \left(\hat{H}_{\mathrm{arb}} x\right)=\left[Q_{j}\left(\frac{x}{m}\right)\right]^{m}+O\left(\frac{|x|^{j+1}}{m^{j}}\right) .
$$

When using the second-order approximation Eq. (B2) in this formula the experimental procedure can be optimized by combining subsequent simulations of $\hat{H}_{1}$ since $\exp \left(\hat{H}_{1} x / 2\right) \exp \left(\hat{H}_{1} x / 2\right)=\exp \left(\hat{H}_{1} x\right)$.
[1] J. G. Bednorz and K. A. Müller, Z. Phys. B: Condens. Matter 64, 189 (1986).

[2] D. N. Basov and T. Timusk, Rev. Mod. Phys. 77, 721 (2005).

[3] M. Greiner, O. Mandel, T. Esslinger, T. W. Hänsch, and I. Bloch, Nature (London) 415, 39 (2002).

[4] M. Greiner, O. Mandel, T. W. Hänsch, and I. Bloch, Nature
(London) 419, 51 (2002).

[5] O. Mandel, M. Greiner, A. Widera, T. Rom, T. W. Hänsch, and I. Bloch, Nature (London) 425, 937 (2003).

[6] O. Mandel, M. Greiner, A. Widera, T. Rom, T. W. Hänsch, and I. Bloch, Phys. Rev. Lett. 91, 010407 (2003).

[7] D. Jaksch, C. Bruder, J. I. Cirac, C. W. Gardiner, and P. Zoller, 
Phys. Rev. Lett. 81, 3108 (1998).

[8] D. Jaksch, H.-J. Briegel, J. I. Cirac, C. W. Gardiner, and P. Zoller, Phys. Rev. Lett. 82, 1975 (1999).

[9] M. W. Zwierlein, J. R. Abo-Shaeer, A. Schirotzek, C. H. Schunck, and W. Ketterle, Nature (London) 435, 1047 (2005).

[10] M. Köhl, H. Moritz, T. Stöferle, K. Günter, and T. Esslinger, Phys. Rev. Lett. 94, 080403 (2005).

[11] D. Jaksch and P. Zoller, Ann. Phys. 315, 52 (2005).

[12] D. Jaksch and P. Zoller, New J. Phys. 5, 56 (2003).

[13] G. Juzeliūnas and P. Öhberg, Phys. Rev. Lett. 93, 033602 (2004).

[14] G. Juzeliūnas, P. Öhberg, J. Ruseckas, and A. Klein, Phys. Rev. A 71, 053614 (2005).

[15] G. Juzeliūnas, J. Ruseckas, P. Öhberg, and M. Fleischhauer, Phys. Rev. A 73, 025602 (2006).

[16] E. J. Mueller, Phys. Rev. A 70, 041603(R) (2004).

[17] A. S. Sørensen, E. Demler, and M. D. Lukin, Phys. Rev. Lett. 94, 086803 (2005).

[18] K. Osterloh, M. Baig, L. Santos, P. Zoller, and M. Lewenstein, Phys. Rev. Lett. 95, 010403 (2005).

[19] J. Ruseckas, G. Juzeliūnas, P. Öhberg, and M. Fleischhauer, Phys. Rev. Lett. 95, 010404 (2005).

[20] F. C. Zhang, Phys. Rev. Lett. 90, 207002 (2003).

[21] E. Jané, G. Vidal, W. Dür, P. Zoller, and J. I. Cirac, Quantum Inf. Comput. 3, 15 (2003).

[22] A. Sørensen and K. Mølmer, Phys. Rev. Lett. 83, 2274 (1999).

[23] L.-M. Duan, E. Demler, and M. D. Lukin, Phys. Rev. Lett. 91, 090402 (2003).

[24] H. F. Trotter, Proc. Am. Math. Soc. 10, 545 (1959).

[25] M. Suzuki, Phys. Lett. A 146, 319 (1990).

[26] M. Suzuki, J. Math. Phys. 32, 400 (1991).

[27] M. Suzuki and T. Yamauchi, J. Math. Phys. 34, 4892 (1993).

[28] M. Suzuki, Commun. Math. Phys. 163, 491 (1994).

[29] E. Dagotto, Rev. Mod. Phys. 66, 763 (1994).

[30] A. Griessner, A. J. Daley, D. Jaksch, and P. Zoller, Phys. Rev. A 72, 032332 (2005).

[31] A. Griessner et al. (in preparation).

[32] P. Anderson, Science 235, 1196 (1987).

[33] J. E. Hirsch, Phys. Rev. Lett. 54, 1317 (1985).

[34] F. C. Zhang and T. M. Rice, Phys. Rev. B 37, R3759 (1988).
[35] A. Damascelli, Z. Hussain, and Z.-X. Shen, Rev. Mod. Phys. 75, 473 (2003).

[36] P. W. Anderson, The Theory of Superconductivity in the High$T_{c}$ Cuprates (Princeton University Press, Princeton, N.J., 1997).

[37] T. Maier, M. Jarrell, T. Pruschke, and M. H. Hettler, Rev. Mod. Phys. 77, 1027 (2005).

[38] H. Eskes and R. Eder, Phys. Rev. B 54, R14226 (1996).

[39] A. Nazarenko, K. J. E. Vos, S. Haas, E. Dagotto, and R. J. Gooding, Phys. Rev. B 51, R8676 (1995).

[40] R. Laughlin, cond-mat/0209269 (unpublished).

[41] B. Paredes and J. I. Cirac, Phys. Rev. Lett. 90, 150402 (2003).

[42] R. C. Ball, Phys. Rev. Lett. 95, 176407 (2005).

[43] F. Verstraete and J. I. Cirac, J. Stat. Mech.: Theory Exp. 2005, P09012.

[44] The experimental realization of such a configuration is planned; I. Bloch, private communication (2005).

[45] D. Jaksch, J. I. Cirac, and P. Zoller, Phys. Rev. A 65, 033625 (2002).

[46] Alternatively, if the real values $t_{r}$ and $U_{r}$ implemented in the lattice are different from the desired values $t$ and $U$, it is also possible to let the system evolve for a real time $\tau_{r}$ such that $\tau t=\tau_{r} t_{r}$ and $\tau U=\tau_{r} U_{r}$.

[47] This method can also be used to simulate negative interaction strengths in Hubbard models in optical lattices. Properly timed periodic raising and lowering of the potential barriers to switch off the hopping terms leads to effective negative onsite interaction strengths.

[48] S. R. Clark and D. Jaksch, Phys. Rev. A 70, 043612 (2004).

[49] E. Altman, E. Demler, and M. D. Lukin, Phys. Rev. A 70, 013603 (2004).

[50] R. Hanbury Brown and R. Q. Twiss, Nature (London) 177, 27 (1956).

[51] S. Fölling, F. Gerbier, A. Widera, O. Mandel, T. Gericke, and I. Bloch, Nature (London) 434, 481 (2005).

[52] D. van Oosten, P. van der Straten, and H. T. C. Stoof, Phys. Rev. A 63, 053601 (2001).

[53] X.-G. Wen and P. A. Lee, Phys. Rev. Lett. 76, 503 (1996).

[54] V. I. Anisimov, M. A. Korotin, I. A. Nekrasov, Z. V. Pchelkina, and S. Sorella, Phys. Rev. B 66, 100502(R) (2002). 